\title{
RESOLVED NEAR-IR SURVEY OF THE INNER GALAXY
}

\author{
M. UNAVANE, G.GILMORE \\ Institute of Astronomy, \\ Cambridge CBз OHA, UK
}

\section{Overview}

The analysis of resolved DENIS (Deep Near Infra Red Survey of the Southern Sky) I,J and $\mathrm{K}$ band data shows good evidence ( $\gtrsim 3 \sigma$ ) in the $\mathrm{J}$ and $\mathrm{K}$ band number counts to support the existence of a kiloparsec-scale bar with its major axis in the first quadrant. A star by star dereddening of this $\mathrm{K}$ data combined with supplementary nbL band data $(3.6 \mu \mathrm{m})$ gives number count contrasts consistent with low angle ( $\lesssim 20$ degrees) bar models in the literature.

\section{Introduction}

There has been recent mounting evidence (Gas kinematics [e.g. Binney et al. 1991], IRAS source asymmetries [e.g. Matsumoto et al. 1982] and surface photometry [e.g. Dwek et al. 1995]) for a kiloparsec scale bar in the centre of our Galaxy. However, the detection of central asymmetries by a resolved stellar survey suffers from the major observational problem of extinction in the disc towards the Galactic bulge. By working in the near infrared, we can reduce the $\sim 40$ magnitudes of visual extinction to $\sim 4$ magnitudes at $\mathrm{K}$ band $(2.2 \mu \mathrm{m})$ and $\sim 2$ magnitudes at $\mathrm{nbL}(3.6 \mu \mathrm{m})$. Observations in several wavebands allow some attempt to be made to disentangle the effects of inhomogeneous dust distribution from the geometry of stellar distributions.

\section{The Data}

We use DENIS data taken within the central $10^{\circ} \times 2^{\circ}$ of the Galactic plane. The processed images were source extracted, and the triplets of IJK images were transformed to fit one another. The total area covered is $17.5 \mathrm{deg}^{2}$, and $\sim 7 \times 10^{5}$ sources are extracted in each band to magnitude limits of 


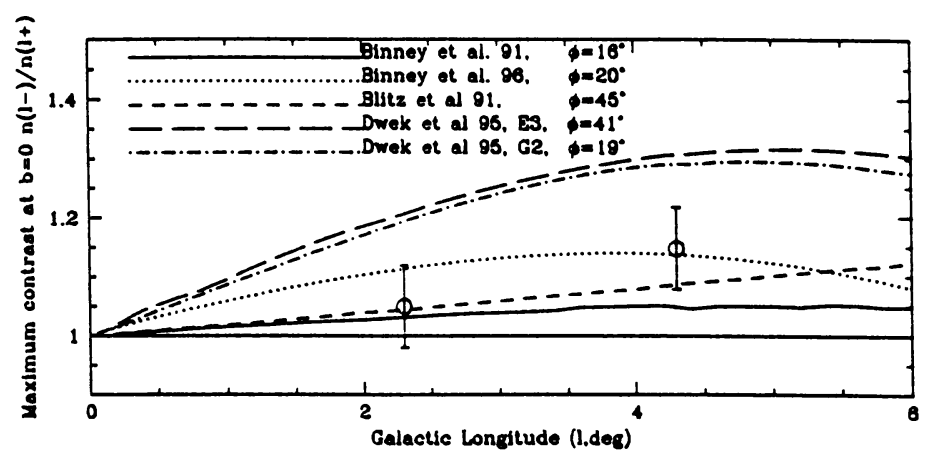

Figure 1. Observed and model bar asymmetries. See text for details.

about 17,15 and 13 in I,J and K. Extensive simulations were carried out to work out the completeness functions for the various colour-magnitude diagrams which were possible. The nbL-band data were taken at UKIRT in July 1996, for regions at $\ell= \pm 2^{\circ}$ and $\pm 4^{\circ}$, at $\mathrm{b}=-0.1^{\circ}$, and covering 277 $\operatorname{arcmin}^{2}$ in total, reaching magnitude 11-12. About $95 \%$ of L-band sources are matched to DENIS K-sources, suggesting that, for observations of the $\mathrm{K}$ and $\mathrm{M}$ giants in the bulge/bar, the extinctive dust is penetrated in both $\mathrm{K}$ and $\mathrm{L}$.

\section{Results}

According to bar models, the greatest asymmetries due to bar structure should occur around $\pm 4^{\circ}$ in longitude (see figure). We construct a simple disk-only model. Using the model and the dust to delimit magnitude ranges where the disk dominates, and where inner Galactic or bulge/bar structure dominates, we find, as expected, that the I band shows no asymmetries in number counts (since, due to the dust, its range is limited to the disk only), a stronger signal of asymmetry in the expected sense in $\mathrm{J}$, and a $>3 \sigma$ detection in the $\mathrm{K}$-band. The combined $\mathrm{K}$ and $\mathrm{nbL}$ band data allow a star-by-star dereddening to be made, which leads to the asymmetry values shown in the figure. The sense of the observed asymmetry is in agreement with the models, with the dynamical (Blitz et al. 1991; Binney et al. 1991) and newest photometric model (Binney et al. 1997) being favoured.

\section{References}

Binney J., Gerhard O.E., Stark A.A., Bally J., Uchida K.I., 1991, MNRAS, 252, 210

Binney J., Gerhard O., Spergel D., 1997, MNRAS, 288, 365

Blitz L., Spergel D.N., 1991, ApJ, 379, 631

Dwek E. et al., 1995, ApJ, 445, 716

Matsumoto T. et al., 1982, in The Galactic Center, AIP Conf. Proc. 83, Riegler G.R., Blandford R.D., eds, AIP, New York, 48 